\title{
Visualization Framework for Computer System Learning
}

\author{
Eiichi Hayakawa ${ }^{1}$, Yuuki Nakagawa ${ }^{2}$, Hideharu Ochiai ${ }^{2}$, \\ Masahiko Fuji ${ }^{2}$, and Yosuke Nishino ${ }^{2}$ \\ ${ }^{1}$ Takushoku University, Faculty of Engineering, \\ Department of Computer Science \\ ${ }^{2}$ Hachioji Soushi High-School \\ hayakawa@cs.takushoku-u.ac.jp, \\ \{Yuuki.Nakagawa, Hideharu.Ochiai, Masahiki.Fuji, \\ Yosuke.Nishino\}@os.cs.takushoku-u.ac.jp
}

\begin{abstract}
In this paper we describe a design of a computer system visualization framework for system software learning. It is difficult for learners to learn the behavior of computer system because of multiple layers of the system and invisibility. For the problem we developed and utilized an integrated learning environment. The results show the problem of high utilization cost of the environment and high development cost of the learning materials. We design a visualization framework based on cloud computing and a material development environment. The framework presents web based visualization component and $\log$ handling facilities. Lecturers can easily develop and provide the visualization material to utilize these facilities for building an appropriate material. In addition learner can use the visualization environment without installing because of running it on the cloud server. This leads to reduce the setup time for the lecturers.
\end{abstract}

\section{Introduction}

It is difficult for computer science learner to study the computer system such as hardware, OS and embedded systems. It is because the learner doesn't trace the behavior of the computer system easily to call each component asynchronously in the multilayer structure. Moreover, the behavior of the system might not effect the operation of the application directly. As a result, it is difficult for the learners to trace the behavior, as they don't understand to process the request from the application and the event from hardware.

To teach the system programming, a lot of teaching materials of making to visible are developed. In the embedded system, the robot device such as LEGO Mindstorms or line tracing cars and evaluation board are often used. Trace log from the devices visualizer [1] is developed for debugging. Our research group has also developed and utilized the integrated learning support environment named Minato [2]. In the Minato the internal behavior of OS and sensor data of the robot are logged and visualized as the state transition of OS processes and the track of sensor data.

In our experience it is apparent that the environment that easily assembles teaching materials is important. Rapid material development environment requires adapting 
various computer systems used in education. The materials easily adaptable to system software education are required because educational system target differs in the university, the enterprise or the carriculum.

In this report we describe a design and a prototype about visualization framework. We present various visualizing components for each layer of the system to assemble these components. Lecturers can create the material for their needs to build the visualization materials through Web browser. Learners can use these components that run on cloud servers in the web browser.

\section{Requirement of the System}

We consider that these requirements are important for system visualization environment through the Minato system development and the experiment in a high school.

(1) Customizability of materials

A development environment on which lecturer customizes materials is required. Our previous environment, Minato, integrates system log monitoring tool, OS or robot visualization tool and system simulator into one application. Learners can use many visualization materials without switching applications. Problems are, however, apparent that it is difficult to allow lecturers to customize the materials where they use OS or embedded system to their needs. In embedded system education every educational institution often utilizes different operating systems such as Android, TOPPERS or eCOS or embedded system such as ARM, MIPS or x86. Material builder requires the environment to easily build materials proper to their environment.

(2) Easily system installation

Easily installation and system management is also required. It is hard to prepare the visualization environment in Java because Java has different virtual machine versions and requires many libraries to install to the local machines. Since various machines such as Windows, Mac or Linux are set up in educational institution, learners are affected with the usability that the OS presented.

The target system requires that it is secure from learner's action and rapid preparation in the classroom. When executing exercise programs on real hardware, inappropriate application or system may crash the system itself and lose system log or tracing data. Moreover, it may be expensive to prepare real hardware to each learner in the classroom.

\section{System Design}

\subsection{System Overall}

Figure 1 shows the overall structure of the system. The system is built from three nodes: Component Node, Execution Node and Service Node.

First, Component Node presents the visualization materials. These are classified into visualizing component and combining component. Visualizing component is minimum component for system program visualization. Combining component is the 


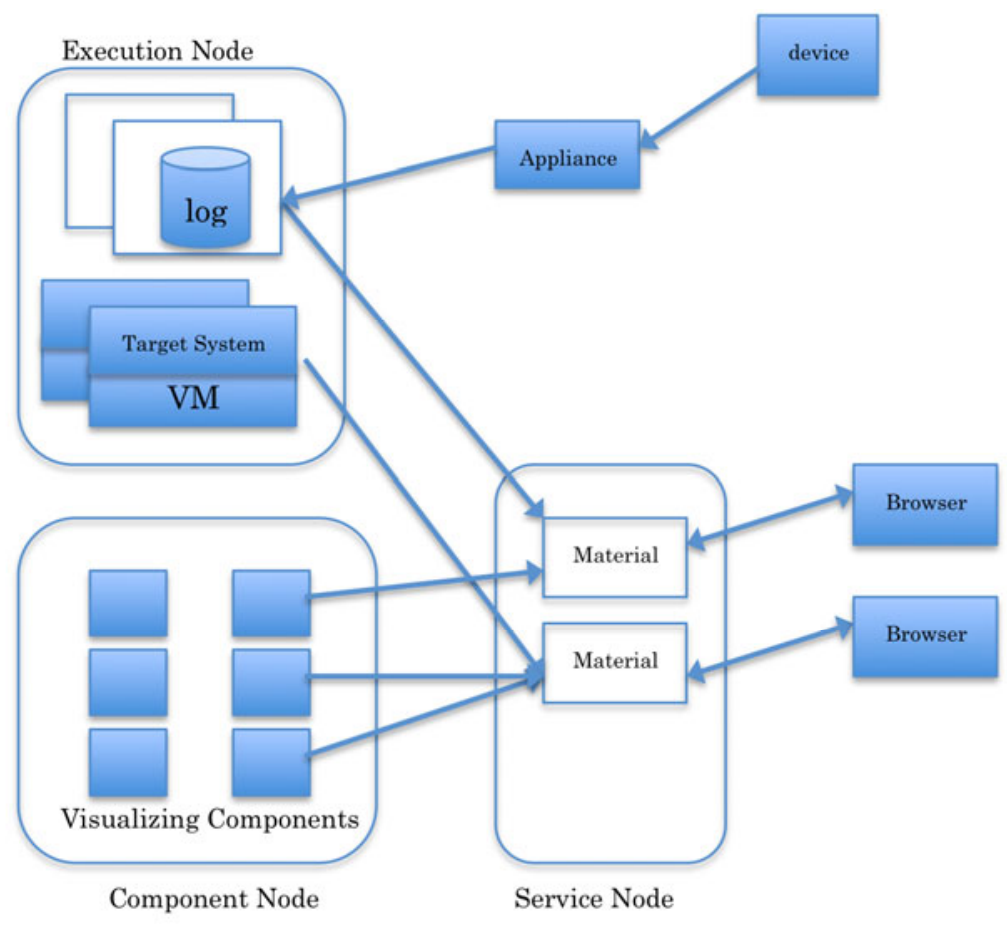

Fig. 1. Overall Structure of the System

aggregate of visualizing components. Lecturers build a material required as a combining component with visualizing components.

Second, Execution Node presents and controls an execution environment for a target system. The environment consists of virtual machine or real hardware system. This node acquires the execution information such as CPU performance, process creation and switch, memory usage and I/O and interrupt information from the target.

Third, Service Node presents materials as assembled components to the learner. This node runs on cloud server and maintains learner's information. Using cloud server, this service can scale out in proportion to increasing learners.

\subsection{Component Node}

Component Node implements in Javascript as jQuery[3] plugin. Common execution control methods such as to control animation speed, replay and step execution are presented as component common methods. The groups of visualization components are following:

- Electrocardiogram chart

- Event list

- State transition chart

- Sequential chart 
- Performance chart

- Architectural chart

- Graph chart

Electrocardiogram chart displays system changes over time such as an electrocardiogram. Event list displays a list about timer or I/O events. Sequential chart also displays these events sequentially. State transition chart displays state transition diagram in the system's behavior such as process, file, etc. Performance chart displays line or bar graphs about system performance. Architectural chart displays hardware components such as CPU, memory, I/O devices. Graph chart displays tree or graph structure to represent the relation among system components.

Assembled component generates a visualization material to assemble visual components. For example, process visualization material is build to assemble Electrocardiogram chart and State transition chart. Electrocardiogram chart represents transitions about processes and State transition chart represents behavior about processes in the OS.

\subsection{Execution Node}

Execution Node is implemented based on KVM [4] kernel virtual machine or QEMU software emulator for hardware not to support KVM. Virtual machine is presented as a node controller of cloud servers to support the increase of users. Real hardware such as evaluation board or robot devices is connected through Execution Node from which components can acquire visualization data. We adopt common visualization data format as JSON because of affinity for Javascript.

Ftrace [5] used in Linux utilizes to acquire log data from OS. It is lower tracing overhead and effect to target system. System information such as process transition, interrupt or $\mathrm{I} / \mathrm{O}$ handling and performance information are acquired through ftrace mechanism.

\subsection{Cloud Server Platform}

Service Node and Execution Node run on node controller in Eucalyptus that is open source cloud computing platform. We can present classroom size visualization materials to increase node controller executing Service Node and Execution Node. We don't require installing Java or visualization application on local machines but only require Web browser. Data from hardware such as robot transfers to the Execution Node through the medium of network appliance. It supports the control and $\log$ data transfer from the device when the hardware doesn't support TCP/IP network.

\section{Prototype System}

Figure 2 shows a prototype material for Android process visualization.

This educational material is build from three components. Upper half component visualizes state transitions about five processes. Lower left visualizes process queues in the Android OS. Green represents running process, yellow represents ready 


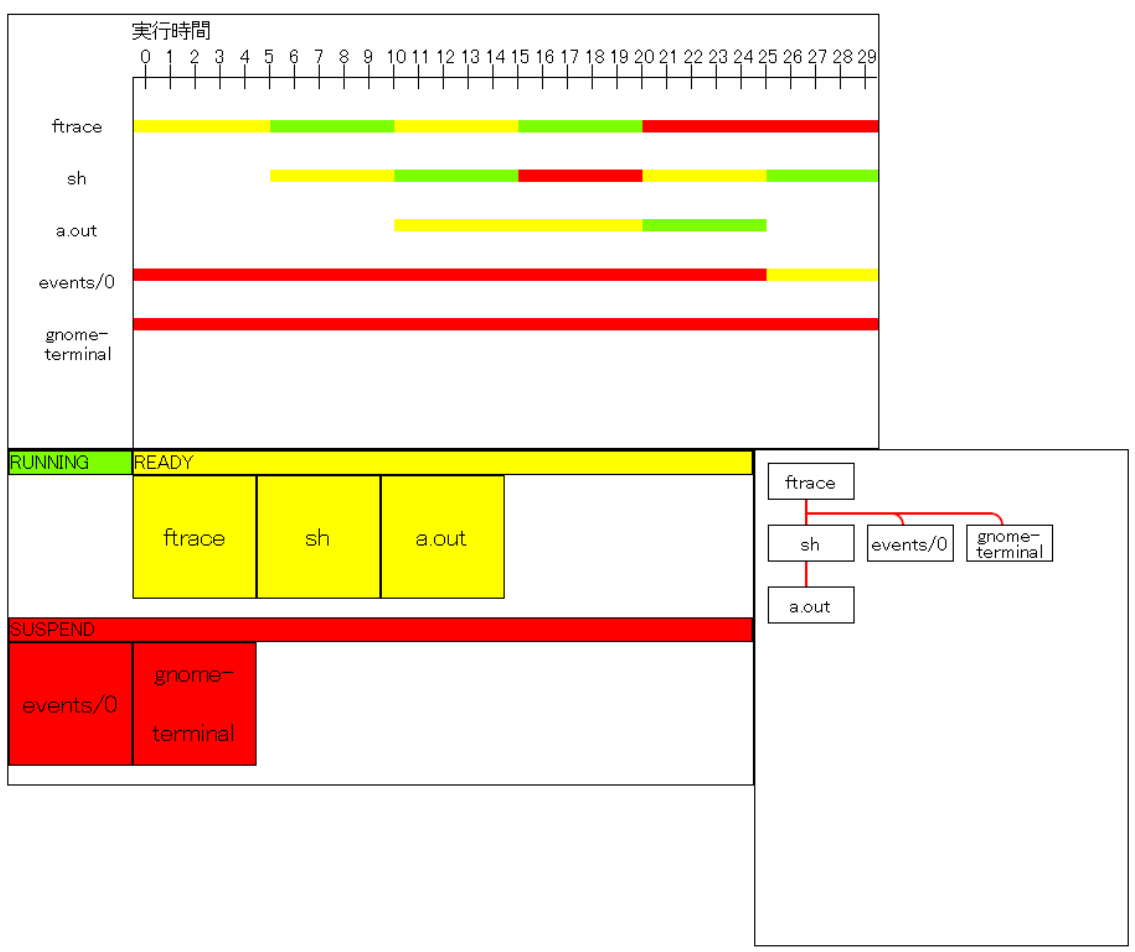

Fig. 2. Prototype of Android visualization tool

processes and red represents suspend processes. Lower right visualizes the process tree of the Android. These components acquire visualization log data from Android simulator or board computer running Android in Execution Node.

Lecturers can add visualization materials not to rewrite the entire program when they require adding it. For example, they add the Javascript code to the assembled component that visualizes process list from log data using event list component when lecturers want to add active process list.

\section{Conclusion}

We describe the design of a computer system visualization framework for system software learning. The framework presents web based visualization materials with three nodes on cloud servers. Lecturers can easily develop and provide the visualization material to utilize these components for building an appropriate material. Running materials on cloud servers, learner is available to use them without installation. This leads to reduce the setup time for the lecturers.

Future work is completely implementing the system. Few components and materials are currently implemented. Previous programs used in the Minato is porting and rewriting to this system. After that we have plan to build the system learning materials using these components and evaluate for high school and university students. 


\section{References}

1. Goto, J., Honda, S., Nagao, T., Takada, H.: Development of Visualization Tool for Trace Log, SIG Technical Reports, 2009-EMB-12, pp. 73-78 (2009) (in Japanese)

2. Nishino, Y., Hayakawa, E.: Minato: Integrated Visualization Environment for Embedded Systems Learning. LNCS, pp. 325-333 (2009)

3. jQuery, http://www.jquery.com/

4. KVM - Kernel Based Virtual Machine

5. http://www. linux-kvm.org/page/Main_Page

6. Ftrace Linux Kernel Tracing

7. http://events.linuxfoundation.org/slides/2010/linuxcon_japan/ linuxcon_jp2010_rostedt.pdf

8. Eucalyptus Systems, http: //www . eucalyptus.com/ 